\title{
Bivariate Mersenne polynomials and matrices
}

\section{Francisco Regis Vieira Alves}

\author{
Department of Mathematics, Institute Federal of Tecnology of State of Ceara (IFCE) \\ Fortaleza-CE, Brazil \\ e-mail: fregis@gmx.fr
}

Received: 7 November 2019

Revised: 19 August 2020

Accepted: 25 August 2020

\begin{abstract}
In this work we present a new notion called bivariate polynomials of Mersenne. We will also discuss certain related matrix properties. Finally, we introduce a notion of the bivariate quaternions of Mersenne.
\end{abstract}

Keywords: Mersenne sequence, Matrix properties, Bivariate Mersenne sequence.

2010 Mathematics Subject Classification: 11B37, 11B39.

\section{Introduction}

The Mersenne sequence is discussed in [1] and [3]. We consider the following set indicating some of these values $\left(0,1,3,7,15,31,63,127,255, \ldots, M_{n}, \ldots\right)$. The Mersenne numbers are defined recurrently by the inhomogeneous equation $M_{n+1}=2 M_{n}+1$. On the other hand, we can write $M_{n+2}=2 M_{n+1}+1$. From these two equations, we can get a homogeneous equation. In fact, we can write $M_{n+2}-M_{n+1}=\left(2 M_{n+1}+1\right)-\left(2 M_{n}+1\right)=2 M_{n+1}-M_{n} \therefore M_{n+2}=3 M_{n+1}-2 M_{n}$.

Definition 1.1. The Mersenne sequence is defined by the recurrence relation:

$$
M_{n+2}=3 M_{n+1}-2 M_{n},
$$

with initial conditions $M_{0}=0, M_{1}=1, n \geqslant 0$.

From the work [1] we know that the roots of the respective characteristic equation $r^{2}-3 r+2=0$ are $r_{1}=2$ and $r_{2}=1$ and we easily get the Binet formula $M_{n}=2^{n}-1$. In the next section, we define the bivariate Mersenne polynomials. Then, after discussing certain properties of matrices, we will present the notion of bivariate quaternions of Mersenne. Other properties of generalized bivariate Fibonacci polynomials and matrices can be found in the work indicated in [2]. 


\section{Bivariate Mersenne polynomials}

We will introduce a new mathematical notion.

Definition 2.1. The bivariate Mersenne polynomials are defined by the recurrence relation:

$$
M_{n+2}(x, y)=3 y M_{n+1}(x, y)-2 x M_{n}(x, y)
$$

with initial conditions $M_{0}(x, y)=0, M_{1}(x, y)=1, n \geqslant 0$.

From Definition 2.1, for the particular case, we can verify that $M_{0}(1,1)=M_{0}$ and $M_{1}(1,1)=M_{1}$. We can determine some particular elements of this polynomial sequence from the Definition 2.1:

$$
\begin{aligned}
M_{2}(x, y) & =3 y \\
M_{3}(x, y) & =9 y^{2}-2 x \\
M_{4}(x, y) & =27 y^{3}-12 x y \\
M_{5}(x, y) & =81 y^{4}-54 x y^{2}+4 x^{2} \\
M_{6}(x, y) & =243 y^{5}-216 x y^{3}+36 x^{2} y \\
M_{7}(x, y) & =729 y^{6}-810 x y^{4}+216 x^{2} y^{2}-8 x^{3} \\
M_{8}(x, y) & =2187 y^{7}-2916 x y^{5}+1080 x^{2} y^{3}-96 x^{3} y \\
M_{9}(x, y) & =6561 y^{8}-10206 x y^{6}+4860 x^{2} y^{4}-720 x^{3} y^{2}+16 x^{4} \\
M_{10}(x, y) & =19683 y^{9}-34992 x y^{7}+20412 x^{2} y^{5}-4320 x^{3} y^{3}+240 x^{4} y
\end{aligned}
$$

etc. In the following theorem we will see the Binet formula for the bivariate Mersenne polynomials.

Theorem 2.2. The Binet's formula for the bivariate Mersenne polynomials is defined by:

$$
M_{n}(x, y)=\frac{r_{1}(x, y)^{n}-r_{2}(x, y)^{n}}{r_{1}(x, y)-r_{1}(x, y)^{n}}
$$

where $r_{1}(x, y)=\frac{3 y+\sqrt{9 y^{2}-8 x}}{2}, r_{2}(x, y)=\frac{3 y-\sqrt{9 y^{2}-8 x}}{2}$ are the roots of the characteristic equation $t^{2}-3 y t+2 x=0$.

Proof. Let us consider the fundamental recurrence relation:

$$
M_{n+2}(x, y)=3 y M_{n+1}(x, y)-2 x M_{n}(x, y) .
$$

We can see that, in the particular case, the roots $r_{1}(x, y)=\frac{3 y+\sqrt{9 y^{2}-8 x}}{2}, r_{2}(x, y)=\frac{3 y-\sqrt{9 y^{2}-8 x}}{2}$ and the characteristc equation $t^{2}-3 y t+2 x=0$, under the condition $9 y^{2}-8 x>0$ result in the original Mersenne sequence. If we take the particular values $x=y=1$, we will find that $r_{1}(1,1)=\frac{3+\sqrt{1}}{2}=r_{1}, r_{2}(1,1)=\frac{3-\sqrt{1}}{2}=r_{2}$ and retrieve the initial characteristic equation of the Mersenne sequence, indicated by $t^{2}-3 t+2=0$. So, in the particular case, we can write $M_{n}(1,1)=\frac{r_{1}(1,1)^{n}-r_{2}(1,1)^{n}}{r_{1}(1,1)-r_{2}(1,1)}=\frac{2^{n}-1^{n}}{2-1}=2^{n}-1=M_{n}$, for every positive integer $n \geqslant 0$. 
Note that using the well-known results involving recursive sequences, the characteristic equation, associated with the recurrence is given by $t^{2}-3 y t+2 x=0$, where the roots are $r_{1}(x, y)=\frac{3 y+\sqrt{9 y^{2}-8 x}}{2}, r_{2}(x, y)=\frac{3 y-\sqrt{9 y^{2}-8 x}}{2}, r_{1}(x, y)+r_{2}(x, y)=3 y, r_{1}(x, y) r_{2}(x, y)=2 x$ or $\frac{1}{r_{1}(x, y)}=\frac{r_{2}(x, y)}{2 x}$.

Moreover, we can easily observe that $r_{1}(x, y)^{2}=\left(3 y r_{1}(x, y)-2 x .1\right)=M_{2}(x, y) r_{1}(x, y)-$ $2 x M_{1}(x, y)$. By mathematical induction, we will consider that $r_{1}(x, y)^{n}=M_{n}(x, y) r_{1}(x, y)-$ $2 x M_{n-1}(x, y)$, for $n \geqslant 0$. Just note that:

$$
\begin{aligned}
r_{1}(x, y)^{n+1} & =r_{1}(x, y)^{n} r_{1}(x, y)=r_{1}(x, y)\left(M_{n}(x, y) r_{1}(x, y)-2 x M_{n-1}(x, y)\right) \\
& =r_{1}(x, y)^{2} M_{n}(x, y)-2 x M_{n-1}(x, y) r_{1}(x, y) \\
& =\left(3 y r_{1}(x, y)-2 x\right) M_{n}(x, y)-2 x M_{n-1}(x, y) r_{1}(x, y) \\
& =3 y r_{1}(x, y) M_{n}(x, y)-2 x M_{n}(x, y)-2 x M_{n-1}(x, y) r_{1}(x, y) \\
& =\left(3 y M_{n}(x, y)-2 x M_{n-1}(x, y)\right) r_{1}(x, y)-2 x M_{n}(x, y) \\
& =M_{n+1}(x, y) r_{1}(x, y)-2 x M_{n}(x, y)=M_{n+2}(x, y) .
\end{aligned}
$$

In general, for $n \geqslant 0$, we can see that $r_{1}(x, y)^{n}=M_{n}(x, y) r_{1}(x, y)-2 x M_{n-1}(x, y)$ and $r_{2}(x, y)^{n}=M_{n}(x, y) r_{2}(x, y)-2 x M_{n-1}(x, y)$. Easily, we will find that $\left(r_{1}(x, y)^{n}-r_{2}(x, y)^{n}\right)=$ $M_{n}(x, y) r_{1}(x, y)-M_{n}(x, y) r_{2}(x, y)=M_{n}(x, y)\left(r_{1}(x, y)-r_{2}(x, y)\right)$.

For the particular case, we can verify that $M_{0}(1,1)=M_{0}$ and $M_{1}(1,1)=M_{1}$. From the recurrence indicated in the Definition 2.1, we can still determine that

$$
\begin{aligned}
& M_{-1}(x, y)=-\frac{1}{2 x}=-\frac{1}{(2 x)^{1}} M_{1}(x, y) \\
& M_{-2}(x, y)=-\frac{3 x}{4 x^{2}}=-\frac{1}{\left(4 x^{2}\right)}(3 y)=-\frac{1}{(2 x)^{2}} M_{2}(x, y) \\
& M_{-3}(x, y)=-\frac{9 y^{2}}{8 x^{3}}+\frac{1}{4 x^{2}}=-\frac{1}{8 x^{3}}\left(9 y^{2}-2 x\right)=-\frac{1}{(2 x)^{3}}=M_{3}(x, y) \\
& M_{-4}(x, y)=-\frac{27 y^{3}}{16 x^{4}}+\frac{3 y}{4 x^{3}}=-\frac{1}{16 x^{4}}\left(27 y^{3}-12 x y\right)=-\frac{1}{(2 x)^{4}} M_{4}(x, y) \\
& M_{-5}(x, y)=-\frac{81 y^{4}}{32 x^{5}}+\frac{27 y^{2}}{16 x^{4}}-\frac{1}{8 x^{3}}=-\frac{1}{32 x^{5}}\left(81 y^{4}-54 x y^{2}+4 x^{2}\right)=-\frac{1}{(2 x)^{5}} M_{5}(x, y) \\
& M_{-6}(x, y)=-\frac{243 y^{5}}{64 x^{6}}+\frac{27 y^{3}}{8 x^{5}}-\frac{9 y}{16 x^{4}}=-\frac{1}{64 x^{6}}\left(243 y^{5}-216 x y^{3}+36 x^{2} y\right)=-\frac{1}{(2 x)^{6}} M_{6}(x, y) \\
& M_{-7}(x, y)=-\frac{729 y^{6}}{128 x^{7}}+\frac{405 y^{4}}{64 x^{6}}-\frac{27 y^{2}}{16 x^{5}}+\frac{1}{16 x^{4}}=-\frac{1}{128 x^{7}}\left(729 y^{6}-810 x y^{4}+216 x^{2} y^{2}-8 x^{3}\right) \\
&=-\frac{1}{(2 x)^{7}} M_{7}(x, y),
\end{aligned}
$$

etc. From these preliminary examples, we demonstrate the next theorem. 
Theorem 2.3. The Binet's formula for the bivariate Mersenne polynomials is defined by:

$$
M_{-n}(x, y)=-\frac{1}{(2 x)^{n}} M_{n}(x, y)
$$

for every positive integer $n \geqslant 0$.

Proof. Since we know that $M_{n}(x, y)=\frac{r_{1}(x, y)^{n}-r_{2}(x, y)^{n}}{r_{1}(x, y)-r_{2}(x, y)}$ and, we replace the convenient index and take:

$$
\begin{aligned}
M_{-n}(x, y) & =\frac{r_{1}(x, y)^{-n}-r_{2}(x, y)^{-n}}{r_{1}(x, y)-r_{2}(x, y)}=\frac{\left(\frac{1}{r_{1}(x, y)}\right)^{n}-\left(\frac{1}{r_{2}(x, y)}\right)^{n}}{r_{1}(x, y)-r_{2}(x, y)}=\frac{\left(\frac{r_{2}(x, y)}{2 x}\right)^{n}-\left(\frac{r_{1}(x, y)}{2 x}\right)^{n}}{r_{1}(x, y)-r_{2}(x, y)} \\
& =\frac{1}{(2 x)^{n}}\left(\frac{\left(r_{2}(x, y)\right)^{n}-\left(r_{1}(x, y)\right)^{n}}{r_{1}(x, y)-r_{2}(x, y)}\right)=-\frac{1}{(2 x)^{n}}\left(\frac{\left(r_{1}(x, y)\right)^{n}-\left(r_{2}(x, y)\right)^{n}}{r_{1}(x, y)-r_{2}(x, y)}\right) \\
& =-\frac{1}{(2 x)^{n}} M_{n}(x, y) .
\end{aligned}
$$

Theorem 2.4. The generating function for the Bivariate Mersenne polynomials is:

$$
G M(t)=\frac{M_{0}(x, y)+\left(M_{1}(x, y)-3 y M_{0}(x, y)\right) t}{1-3 y t+2 x t^{2}}=\frac{t}{1-3 y t+2 x t^{2}} .
$$

Proof. We consider the formal series indicated by $G M(t)=\sum_{i=0}^{\infty} M_{i}(x, y) t^{i}$, where the coefficients are exactly the bivariate Mersenne polynomials. We recall, however, the following relationship and we replace the convenient index and take

$$
M_{n+2}(x, y)-3 y M_{n+1}(x, y)+2 x M_{n}(x, y)=0, \forall n \geqslant 0 .
$$

In this way, we will consider the following expressions constituted as infinite sums:

$$
\begin{aligned}
1 G M(t) & =M_{0}(x, y) t^{0}+M_{1}(x, y) t+M_{2}(x, y) t^{2}+\ldots+M_{n}(x, y) t^{n}+\ldots \\
(-3 y) G M(t) t & =-3 y M_{0}(x, y) t-3 y M_{1}(x, y) t^{2}-3 y M_{2}(x, y) t^{3}-\ldots-3 y M_{n-1}(x, y) t^{n}-\ldots \\
(2 x) G M(t) t^{2} & =2 x M_{0}(x, y) t^{2}+2 x M_{1}(x, y) t^{3}+2 x M_{2}(x, y) t^{4}+\ldots+2 x M_{n-2}(x, y) t^{n}+\ldots
\end{aligned}
$$

Then, we group the expression:

$$
\begin{aligned}
& G M(t)-3 y G M(t) t+2 x G M(t) t^{2} \\
&= G M(t)\left(1-3 y t+2 x t^{2}\right) \\
&= M_{0}(x, y)+\left(M_{1}(x, y)-3 y M_{0}(x, y)\right) t+\left(M_{2}(x, y)-3 y M_{1}(x, y)+2 x M_{0}(x, y)\right) t^{2} \\
&+0 t^{3}+0 t^{4}+\ldots+\left(M_{n}(x, y)-3 y M_{n-1}(x, y)+2 x M_{n-2}(x, y)\right) t^{n}+\ldots
\end{aligned}
$$

Finally, we will find that

$$
G M(t)\left(1-3 y t+2 x t^{2}\right)=M_{0}(x, y)+\left(M_{1}(x, y)-3 y M_{0}(x, y)\right) t
$$

or, we can write the generating function

$$
G M(t)=\frac{M_{0}(x, y)+\left(M_{1}(x, y)-3 y M_{0}(x, y) t\right.}{1-3 y t+2 x t^{2}}=\frac{t}{1-3 y t+2 x t^{2}} .
$$


Let us now look at some classic identities.

Theorem 2.5. (Catalan's identity) For $n \geqslant r$, we have:

(i) $M_{n-r}(x, y) M_{n+r}(x, y)-M_{n}(x, y)^{2}=(2 x)^{n}\left(\frac{2-r_{1}(x, y)^{-r} r_{2}(x, y)^{r}-r_{2}(x, y)^{-r} r_{1}(x, y)^{r}}{r_{1}(x, y)-r_{2}(x, y)}\right)$;

(ii) (Cassini's identity) $M_{n-1}(x, y) M_{n+1}(x, y)-M_{n}(x, y)^{2}=(2 x)^{n-1}\left(\frac{4 x-r_{2}(x, y)^{2}-r_{1}(x, y)^{2}}{r_{1}(x, y)-r_{2}(x, y)}\right)$.

Proof. Immediately, we consider the expression:

$$
\begin{aligned}
& M_{n-r}(x, y) M_{n+r}(x, y)-M_{n}(x, y)^{2} \\
&=\left(\frac{r_{1}(x, y)^{n-r}-r_{2}(x, y)^{n-r}}{r_{1}(x, y)-r_{2}(x, y)}\right)\left(\frac{r_{1}(x, y)^{n+r}-r_{2}(x, y)^{n+r}}{r_{1}(x, y)-r_{2}(x, y)}\right) \\
&-\left(\frac{r_{1}(x, y)^{n}-r_{2}(x, y)^{n}}{r_{1}(x, y)-r_{2}(x, y)}\right)\left(\frac{r_{1}(x, y)^{n}-r_{2}(x, y)^{n}}{r_{1}(x, y)-r_{2}(x, y)}\right) \\
&=\left(\frac{r_{1}(x, y)^{n-r} r_{1}(x, y)^{n+r}-r_{1}(x, y)^{n-r} r_{2}(x, y)^{n+r}-r_{2}(x, y)^{n-r} r_{1}(x, y)^{n+r}+r_{2}(x, y)^{n-r} r_{2}(x, y)^{n+r}}{r_{1}(x, y)-r_{2}(x, y)}\right) \\
&-\frac{\left(r_{1}(x, y)^{n}-r_{2}(x, y)^{n}\right)^{2}}{r_{1}(x, y)-r_{2}(x, y)} \\
&= \frac{r_{1}(x, y)^{2 n}+r_{2}(x, y)^{2 n}-\left(r_{1}(x, y) r_{2}(x, y)\right)^{n} r_{1}(x, y)^{-r} r_{2}(x, y)^{r}-\left(r_{2}(x, y) r_{1}(x, y)\right)^{n} r_{2}(x, y)^{-r} r_{1}(x, y)^{r}}{r_{1}(x, y)-r_{2}(x, y)} \\
&-\frac{\left.\left(r_{1}(x, y)\right)^{n}-r_{2}(x, y)^{n}\right)^{2}}{r_{1}(x, y)-r_{2}(x, y)} \\
&= \frac{r_{1}(x, y)^{2 n}+r_{2}(x, y)^{2 n}-(2 x)^{n} r_{1}(x, y)^{-1}-(2 x)^{n} r_{2}(x, y)^{-r} r_{1}(x, y)-\left(r_{1}(x, y)^{n}-r_{2}(x, y)^{n}\right)^{2}}{r_{1}(x, y)-r_{2}(x, y)} \\
&= \frac{r_{1}(x, y)^{2 n}+r_{2}(x, y)^{2 n}-(2 x)^{n} r_{1}(x, y)^{-r} r_{2}(x, y)^{r}-(2 x)^{n} r_{2}(x, y)^{-r} r_{1}(x, y)^{r}-r_{1}(x, y)^{2 n}-r_{2}(x, y)^{2 n}}{r_{1}(x, y)-r_{2}(x, y)} \\
&+\frac{2 r_{1}(x, y)^{n} r_{2}(x, y)^{n}}{r_{1}(x, y)-r_{2}(x, y)} \\
&= \frac{2(2 x)^{n}-(2 x)^{n} r_{1}(x, y)^{-r} r_{2}(x, y)^{r}-(2 x)^{n} r_{2}(x, y)^{-r} r_{1}(x, y)^{r}}{r_{1}(x, y)-r_{2}(x, y)} \\
&=(2 x)^{n}\left(\frac{2-r_{1}(x, y)^{-r} r_{2}(x, y)^{r}-r_{2}(x, y)^{-r} r_{1}(x, y)^{r}}{r_{1}(x, y)-r_{2}(x, y)} .\right.
\end{aligned}
$$

For the particular case, if we take $r=1$ we will find that:

$$
\begin{aligned}
M_{-1}(x, y) M_{n+1}(x, y)-M_{n}(x, y)^{2} & =(2 x)^{n}\left(\frac{2-r_{1}(x, y)^{-1} r_{2}(x, y)-r_{2}(x, y)^{-1} r_{1}(x, y)}{r_{1}(x, y)-r_{2}(x, y)}\right) \\
& =(2 x)^{n}\left(\frac{\left.2-\frac{r_{2}(x, y)}{r_{1}(x, y)}-\frac{r_{1}(x, y)}{r_{2}(x, y)}\right)}{r_{1}(x, y)-r_{2}(x, y)}\right) \\
& =(2 x)^{n}\left(\frac{2\left(r_{1}(x, y) r_{2}(x, y)\right)-r_{2}(x, y)^{2}-r_{1}(x, y)^{2}}{\left(r_{1}(x, y) r_{2}(x, y)\right)\left(r_{1}(x, y)-r_{2}(x, y)\right)}\right) \\
& =(2 x)^{n}\left(\frac{4 x-r_{2}(x, y)^{2}-r_{1}(x, y)^{2}}{2 x\left(r_{1}(x, y)-r_{2}(x, y)\right)}\right) \\
& =(2 x)^{n-1}\left(\frac{4 x-r_{2}(x, y)^{2}-r_{1}(x, y)^{2}}{r_{1}(x, y)-r_{2}(x, y)}\right),
\end{aligned}
$$

follows the result in the second item. 
In the following section, we will introduce some matrix properties related to the bivariate Mersenne polynomials and their generating matrix.

\section{Some matrix properties of the Mersenne polynomials}

In this section we will study the properties of the powers of the following matrices defined by $B(x, y)=\left(\begin{array}{cc}0 & 1 \\ -2 x .1 & 3 y\end{array}\right)$ and $B(x, y)^{-1}=\left(\begin{array}{cc}\frac{3 y}{2 x} & -\frac{1}{2 x} 1 \\ 1 & 0\end{array}\right)$.

Let us start by analyzing the behavior of the following matrix powers

$$
B(x, y)^{n}=\left(\begin{array}{cc}
0 & 1 \\
-2 x .1 & 3 y
\end{array}\right)^{n}
$$

for $n \geqslant 1$ :

$$
\begin{aligned}
& B(x, y)=\left(\begin{array}{cc}
0 & 1 \\
-2 x .1 & 3 y
\end{array}\right)=\left(\begin{array}{ll}
-2 x M_{0}(x, y) & M_{1}(x, y) \\
-2 x M_{1}(x, y) & M_{2}(x, y)
\end{array}\right) \\
& B(x, y)^{2}=\left(\begin{array}{cc}
-2 x & 3 y \\
-2 x(3 y) & 9 y^{2}-2 x
\end{array}\right)=\left(\begin{array}{cc}
-2 x M_{1}(x, y) & M_{2}(x, y) \\
-2 x M_{2}(x, y) & M_{3}(x, y)
\end{array}\right), \\
& B(x, y)^{3}=\left(\begin{array}{cc}
-2 x(3 y) & 9 y^{2}-2 x \\
-2 x\left(9 y^{2}-2 x\right) & 27 y^{3}-12 x y
\end{array}\right)=\left(\begin{array}{cc}
-2 x M_{2}(x, y) & M_{3}(x, y) \\
-2 x M_{3}(x, y) & M_{4}(x, y)
\end{array}\right), \\
& B(x, y)^{4}=\left(\begin{array}{cc}
-2 x\left(9 y^{2}-2 x\right) & 27 y^{3}-12 x y \\
-2 x\left(27 y^{3}-12 x y\right) & 81 y^{4}-54 x y^{2}+4 x^{2}
\end{array}\right)=\left(\begin{array}{cc}
-2 x M_{3}(x, y) & M_{4}(x, y) \\
-2 x M_{4}(x, y) & M_{5}(x, y)
\end{array}\right), \\
& B(x, y)^{5}=\left(\begin{array}{cc}
-2 x\left(27 y^{3}-12 x y\right) & 81 y^{4}-54 x y^{2}+4 x^{2} \\
-2 x\left(81 y^{4}-54 x y^{2}+4 x^{2}\right) & 243 y^{5}-216 x y^{3}+24 x^{2} y
\end{array}\right) \\
& =\left(\begin{array}{ll}
-2 x M_{4}(x, y) & M_{5}(x, y) \\
-2 x M_{5}(x, y) & M_{6}(x, y)
\end{array}\right), \\
& B(x, y)^{6}=\left(\begin{array}{cc}
-2 x\left(81 y^{4}-54 x y^{2}+4 x^{2}\right) & 243 y^{5}-216 x y^{3}+36 x^{2} y \\
-2 x\left(243 y^{5}-216 x y^{3}+36^{2} y\right) & 729 y^{6}-810 x y^{4}+216 x^{2} y^{2}-8 x^{3}
\end{array}\right) \\
& =\left(\begin{array}{ll}
-2 x M_{5}(x, y) & M_{6}(x, y) \\
-2 x M_{6}(x, y) & M_{7}(x, y)
\end{array}\right) \text {. }
\end{aligned}
$$

From these particular examples, let us define the following matrix:

$$
B_{n}(x, y)=\left(\begin{array}{cc}
-2 x M_{n-1}(x, y) & M_{n}(x, y) \\
-2 x M_{n}(x, y) & M_{n+1}(x, y)
\end{array}\right),
$$

for every integer $n \geqslant 0$. 
Theorem 3.1. For every integers $n, m \geqslant 0$, we have:

(i) $B(x, y)^{n}=\left(\begin{array}{cc}0 & 1 \\ -2 x .1 & 3 y\end{array}\right)=\left(\begin{array}{cc}-2 x M_{n-1}(x, y) & M_{n}(x, y) \\ -2 x M_{n}(x, y) & M_{n+1}(x, y)\end{array}\right)=B_{n}(x, y)$;

(ii) $\operatorname{det}(B(x, y))=\operatorname{det}\left(B(x, y)^{n}\right)=\operatorname{det}\left(\begin{array}{cc}0 & 1 \\ -2 x & 3 y\end{array}\right)^{n}=2^{n} x^{n}$;

(iii) $M_{n-1}(x, y) M_{n+1}(x, y)-M_{n}(x, y)^{2}=-2 x^{n-1} x^{n-1}$;

(iv) $B_{n+m}(x, y)=B_{n}(x, y) B_{m}(x, y)=B_{m}(x, y) B_{n}(x, y)$.

Proof. By mathematical induction, for $n, m>0$, we will consider the power:

$$
B(x, y)^{n}=\left(\begin{array}{cc}
0 & 1 \\
-2 x .1 & 3 y
\end{array}\right)^{n}=\left(\begin{array}{cc}
-2 x M_{n-1}(x, y) & M_{n}(x, y) \\
-2 x M_{n}(x, y) & M_{n+1}(x, y)
\end{array}\right) .
$$

At the next step, we take:

$$
\begin{aligned}
B(x, y)^{n+1} & =B(x, y)^{n} B(x, y)=\left(\begin{array}{cc}
-2 x M_{n-1}(x, y) & M_{n}(x, y) \\
-2 x M_{n}(x, y) & M_{n+1}(x, y)
\end{array}\right)\left(\begin{array}{cc}
0 & 1 \\
-2 x .1 & 3 y
\end{array}\right) \\
& =\left(\begin{array}{cc}
-2 x M_{n}(x, y) & 3 y M_{n}(x, y)-2 x M_{n-1}(x, y) \\
-2 x M_{n+1}(x, y) & 3 y M_{n+1}(x, y)-2 x M_{n}(x, y)
\end{array}\right) \\
& =\left(\begin{array}{cc}
-2 x M_{n}(x, y) & M_{n+1}(x, y) \\
-2 x M_{n+1}(x, y) & M_{n+2}(x, y)
\end{array}\right) \\
& =B_{n+1}(x, y),
\end{aligned}
$$

for every integer $n \geqslant 0$. From this, we can determine that

$$
\begin{aligned}
\operatorname{det}\left(B_{n}(x, y)\right)=\operatorname{det}\left(B(x, y)^{n}\right) & =\operatorname{det}\left(\begin{array}{cc}
-2 x M_{n-1}(x, y) & M_{n}(x, y) \\
-2 x M_{n}(x, y) & M_{n+1}(x, y)
\end{array}\right) \\
& =\operatorname{det}\left(\begin{array}{cc}
0 & 1 \\
-2 x .1 & 3 y
\end{array}\right)^{n}=(2 x)^{n} .
\end{aligned}
$$

However, we can verify that $(-2 x)\left(M_{n-1}(x, y) M_{n+1}(x, y)-M_{n}(x, y)^{2}\right)=2^{n} x^{n}$.

Thus, we can find that $(-2 x) .\left(M_{n-1}(x, y) M_{n+1}(x, y)-M_{n}(x, y)^{2}\right)=2^{n} x^{n}$ for every integer $n \geqslant 0$.

Finally, we verify that $B_{n+m}(x, y)=B(x, y)^{n+m}=B(x, y)^{n} B(x, y)^{m}=B(x, y)^{m} B(x, y)^{n}$ $=B_{m+n}(x, y)$, for every positive integers $n, m$.

Let us define the following matrix

$$
B_{-n}(x, y)=\left(\begin{array}{cc}
-2 x M_{-n-1}(x, y) & M_{-n}(x, y) \\
-2 x M_{-n}(x, y) & M_{-n+1}(x, y)
\end{array}\right)
$$

for $n \geqslant 1$.

We will now check some properties of the matrix powers $\left(\begin{array}{cc}0 & 1 \\ -2 x .1 & 3 y\end{array}\right)^{-n}$. 
Theorem 3.2. For every integers $n>0, m>0$, we have:

(i) $B_{-n}(x, y)=\left(\begin{array}{cc}-2 x M_{-(n+1)}(x, y) & M_{-(n)}(x, y) \\ -2 x M_{-(n)}(x, y) & M_{-(n-1)}(x, y)\end{array}\right)=\left(B_{n}(x, y)^{-1}\right)=\left(\begin{array}{cc}0 & 1 \\ -2 x .1 & 3 y\end{array}\right)^{-n}$;

(ii) $\operatorname{det}\left(B_{-n}(x, y)\right)=\operatorname{det}\left(B_{n}(x, y)^{-1}\right)=\frac{1}{(2 x)^{n}}$;

(iii) $B_{-(n+m)}(x, y)=B_{-n}(x, y) B_{-m}(x, y)=B_{-m}(x, y) B_{-n}(x, y)$.

Proof. From Theorem 2.2 we know that $M_{-n}(x, y)=-\frac{1}{(2 x)^{n}} M_{n}(x, y)$. Now, we consider the matrix:

$$
\begin{aligned}
B_{-n}(x, y) & =\left(\begin{array}{cc}
-2 x M_{-n-1}(x, y) & M_{-n}(x, y) \\
-2 x M_{-n}(x, y) & M_{-n+1}(x, y)
\end{array}\right)=\left(\begin{array}{cc}
-2 x M_{-(n+1)}(x, y) & M_{-n}(x, y) \\
-2 x M_{-n}(x, y) & M_{-(n-1)}(x, y)
\end{array}\right) \\
& =\left(\begin{array}{cc}
-2 x\left(-\frac{1}{(2 x)^{n+1}} M_{n+1}(x, y)\right) & -\frac{1}{(2 x)^{n}} M_{n}(x, y) \\
-2 x\left(-\frac{1}{(2 x)^{n}} M_{n}(x, y)\right) & -\frac{1}{(2 x)^{n-1}} M_{n-1}(x, y)
\end{array}\right) \\
& =\left(\begin{array}{cc}
\frac{1}{(2 x)^{n}} M_{n+1}(x, y) & -\frac{1}{(2 x)^{n}} M_{n}(x, y) \\
2 x\left(-\frac{1}{(2 x)^{n}} M_{n}(x, y)\right) & -\frac{2 x}{(2 x)^{n}} M_{n-1}(x, y)
\end{array}\right) \\
& =\frac{1}{(2 x)^{n}}\left(\begin{array}{ll}
M_{n+1}(x, y) & -M_{n}(x, y) \\
2 x M_{n}(x, y) & -2 x M_{n-1}(x, y)
\end{array}\right) \\
& =\left(B_{n}(x, y)\right)^{-1} .
\end{aligned}
$$

Immediately, from the equality established in the previous item, it follows that

$$
\operatorname{det}\left(B_{-n}(x, y)\right)=\operatorname{det}\left(B_{n}(x, y)\right)^{-1}=\frac{1}{(2 x)^{n}},
$$

for every integer $n \geqslant 0$. Moreover, repeating the above arguments, we can determine that:

$$
\begin{aligned}
B_{-(n+m)}(x, y) & =(B(x, y))^{-(n+m)}=(B(x, y))^{-n}(B(x, y))^{-m} \\
& =B_{-n}(x, y) B_{-m}(x, y)=B_{-m}(x, y) B_{-n}(x, y) .
\end{aligned}
$$

We can observe some particular cases for $B(x, y)^{-n}$, as follows.

$$
\begin{aligned}
& B(x, y)^{-1}=\left(\begin{array}{cc}
\frac{3 y}{2 x} & -\frac{1}{2 x} 1 \\
1 & 0
\end{array}\right)=\left(\begin{array}{cc}
-2 x\left(-\frac{3 y}{2 x^{2}}\right) & -\frac{1}{2 x} 1 \\
-2 x & 0
\end{array}\right)=\left(\begin{array}{ll}
-2 x M_{-2}(x, y) & M_{-1}(x, y) \\
-2 x M_{-1}(x, y) & M_{0}(x, y)
\end{array}\right), \\
& B(x, y)^{-2}=\left(\begin{array}{cc}
\frac{9 y^{2}-2 x}{4 x^{2}} & -\frac{3 y}{4 x^{2}} \\
\frac{3 y}{2 x} & -\frac{1}{2 x}
\end{array}\right)=\left(\begin{array}{cc}
-2 x\left(-\frac{-9 y^{2}+2 x}{8 x^{3}}\right) & -\frac{3 y}{4 x^{2}} \\
\frac{3 y}{2 x} & -\frac{1}{2 x}
\end{array}\right)=\left(\begin{array}{ll}
-2 x M_{-3}(x, y) & M_{-2}(x, y) \\
-2 x M_{-2}(x, y) & M_{-1}(x, y)
\end{array}\right), \\
& B(x, y)^{-3}=\left(\begin{array}{cc}
\frac{9 y^{2}-2 x}{4 x^{2}} & -\frac{1}{8} \frac{\left(9 y^{2}-2 x\right)}{x^{3}} \\
\frac{1}{4} \frac{\left.9 y^{2}-2 x\right)}{x^{2}} & -\frac{3 y}{4 x^{2}}
\end{array}\right)=\left(\begin{array}{cc}
-2 x M_{-4}(x, y) & M_{-3}(x, y) \\
-2 x M_{-3}(x, y) & M_{-2}(x, y)
\end{array}\right) \\
& B(x, y)^{-4}=\left(\begin{array}{cc}
\frac{81 y^{4}}{16 x^{4}}-\frac{27 y^{2}}{8 x^{3}}+\frac{1}{4 x^{2}} & -\frac{27 y^{3}}{16 x^{4}}+\frac{3 y}{4 x^{3}} \\
-2 x\left(-\frac{27 y^{3}}{16 x^{4}}+\frac{3 y}{4 x^{3}}\right. & -\frac{9 y^{2}}{8 x^{3}}+\frac{1}{4 x^{2}}
\end{array}\right)=\left(\begin{array}{ll}
-2 x M_{-5}(x, y) & M_{-4}(x, y) \\
-2 x M_{-4}(x, y) & M_{-3}(x, y)
\end{array}\right),
\end{aligned}
$$




$$
\begin{aligned}
B(x, y)^{-5} & =\left(\begin{array}{cc}
\frac{243 y^{5}}{32 x^{5}}-\frac{27 y^{3}}{4 x^{4}}+\frac{9 y}{8 x^{3}} & -\frac{81 y^{4}}{32 x^{4}}+\frac{27 y^{2}}{16 x^{4}}-\frac{1}{8 x^{3}} \\
-2 x\left(-\frac{81 y^{4}}{32 x^{4}}+\frac{27 y^{2}}{16 x^{4}}\right)-\frac{1}{8 x^{3}} & -\frac{9 y^{2}}{8 x^{3}}+\frac{1}{4 x^{2}}
\end{array}\right) \\
& =\left(\begin{array}{ll}
-2 x M_{-6}(x, y) & M_{-5}(x, y) \\
-2 x M_{-5}(x, y) & M_{-4}(x, y)
\end{array}\right) .
\end{aligned}
$$

In the last section we present the notion of Merssene's bivariate quaternions. Here we verify the behavior of particular cases that prove the Theorem 3.2.

\section{Quaternion Bivariate of Mersenne}

In this section we will define the Mersenne quaternion polynomials. The general case is discussed in the work [3].

Definition 4.1. The nth Mersenne quaternion bivariate polynomial is defined by $Q M_{n}(x, y)=$ $M_{n}(x, y)+M_{n+1}(x, y) i+M_{n+2}(x, y) j+M_{n+3}(x, y) k$, where the canonical basis $\{1, \vec{i}, \vec{j}, \vec{k}\}$. The quaternion multiplication is defined by the following formal rules $i^{2}=j^{2}=k^{2}=i j k=-1$.

We can verify some examples:

$$
\begin{aligned}
Q M_{-1}(x, y) & =-\frac{1}{2 x}+0 i+1 j+3 y k, \\
Q M_{0}(x, y) & =0+i+3 y j+\left(9 y^{2}-2 x\right) k, \\
Q M_{1}(x, y) & =1+3 y i+\left(9 y^{2}-2 x\right) j+\left(27 y^{3}-12 x y\right) k,
\end{aligned}
$$

etc. Let us look at some preliminary properties.

Moreover, we can take the

$$
Q M_{-n}(x, y)=M_{-n}(x, y)+M_{-n+1}(x, y) i+M_{n+2}(x, y) j+M_{-n+3}(x, y) k
$$

and, since we know evaluate the terms $M_{-n}(x, y)=-\frac{1}{(2 x)^{n}} M_{n}(x, y)$, we can determine:

$$
\begin{aligned}
& Q M_{-1}(x, y)=M_{-1}(x, y)+M_{0}(x, y) i+M_{1}(x, y) j+M_{2}(x, y) k \\
& Q M_{-2}(x, y)=M_{-2}(x, y)+M_{-1}(x, y) i+M_{0}(x, y) j+M_{1}(x, y) k \\
& Q M_{-3}(x, y)=M_{-3}(x, y)+M_{-2}(x, y) i+M_{-1}(x, y) j+M_{0}(x, y) k,
\end{aligned}
$$

etc.

Theorem 4.2. For every integer $n \geqslant 0$, we have:

(i) $Q M_{n+2}(x, y)=3 y Q M_{n+1}(x, y)-2 x Q M_{n}(x, y)$;

(ii) $Q M_{n}(x, y)=\frac{p(x, y) r_{1}(x, y)^{n}-q(x, y) r_{2}(x, y)^{n}}{r_{1}(x, y)-r_{2}(x, y)}$,

$$
\begin{aligned}
& p(x, y)=1+r_{1}(x, y) i+r_{1}(x, y)^{2} j+r_{1}(x, y)^{3} k, \\
& q(x, y)=1+r_{2}(x, y) i+r_{2}(x, y)^{2} j+r_{2}(x, y)^{3} k ;
\end{aligned}
$$


(iii) $Q M_{-n}(x, y)=\frac{1}{(2 x)^{n}} \frac{p(x, y) r_{2}(x, y)-q(x, y) r_{1}(x, y)^{n}}{r_{1}(x, y)-r_{2}(x, y)}$.

Proof. We can observe directly, developing the following expression:

$$
\begin{aligned}
3 y Q M_{n+1}(x, y)-2 x Q M_{n}(x, y)= & \left(3 y M_{n+1}(x, y)+3 y M_{n+2}(x, y) i+3 y M_{n+3}(x, y) j+3 y M_{n+4}(x, y) k\right) \\
& -2 x M_{n}(x, y)-2 x M_{n+1}(x, y) i-2 x M_{n+2}(x, y) j-2 x M_{n+3}(x, y) k \\
= & \left(3 y M_{n+1}(x, y)-2 x M_{n}(x, y)\right)+\left(3 y M_{n+2}(x, y)-2 x M_{n+1}(x, y)\right) i \\
& +\left(3 y M_{n+3}(x, y)-2 x M_{n+2}(x, y)\right) j+\left(3 y M_{n+4}(x, y)-2 x M_{n+3}(x, y)\right) k \\
= & M_{n+2}(x, y)+M_{n+3}(x, y) i+M_{n+4}(x, y) j+M_{n+5}(x, y) k \\
= & Q M_{n+2}(x, y) .
\end{aligned}
$$

From the previous definition, since we know the Binet's formula, we write:

$$
\begin{aligned}
Q M_{n}(x, y)= & \frac{r_{1}(x, y)^{n}-r_{2}(x, y)^{n}}{r_{1}(x, y)-r_{2}(x, y)}+\frac{r_{1}(x, y)^{n+1}-r_{2}(x, y)^{n+1}}{r_{1}(x, y)-r_{2}(x, y)} i+\frac{r_{1}(x, y)^{n+2}-r_{2}(x, y)^{n+2}}{r_{1}(x, y)-r_{2}(x, y)} j \\
& +\frac{r_{1}(x, y)^{n+3}-r_{2}(x, y)^{n+3}}{r_{1}(x, y)-r_{2}(x, y)} k \\
= & \frac{\left(r_{1}(x, y)^{n}+r_{1}(x, y)^{n+1} i+r_{1}(x, y)^{n+2} j+r_{1}(x, y)^{n+3} k\right)-\left(r_{2}(x, y)^{n}+r_{2}(x, y)^{n+1} i+r_{2}(x, y)^{n+2} j\right)}{r_{1}(x, y)-r_{2}(x, y)} \\
& +\frac{\left(r_{1}(x, y)^{n+3} k\right)-\left(r_{2}(x, y)^{n}+r_{2}(x, y)^{n+1} i+r_{2}(x, y)^{n+2} j+r_{2}(x, y)^{n+3} k\right)}{r_{1}(x, y)-r_{2}(x, y)} \\
= & \frac{r_{1}(x, y)^{n}\left(1+r_{1}(x, y) i+r_{1}(x, y)^{2} j+r_{1}(x, y)^{3} k\right)-r_{2}(x, y)^{n}\left(1+r_{2}(x, y) i+r_{2}(x, y)^{2} j+r_{2}(x, y)^{3} k\right)}{r_{1}(x, y)-r_{2}(x, y)} \\
= & \frac{p(x, y) r_{1}(x, y)^{n}-q(x, y) r_{2}(x, y)^{n}}{r_{1}(x, y)-r_{2}(x, y)},
\end{aligned}
$$

where

$$
p(x, y)=1+r_{1}(x, y) i+r_{1}(x, y)^{2} j+r_{1}(x, y)^{3} k
$$

and

$$
q(x, y)=1+r_{2}(x, y) i+r_{2}(x, y)^{2} j+r_{2}(x, y)^{3} k .
$$

Finally, we will replace the convenient index

$$
\begin{aligned}
Q M_{-n}(x, y) & =\frac{p(x, y) r_{1}(x, y)^{-n}-q(x, y) r_{2}(x, y)^{-n}}{r_{1}(x, y)-r_{2}(x, y)} \\
& =\frac{p(x, y)\left(\frac{1}{r_{1}(x, y)}\right)^{n}-q(x, y)\left(\frac{1}{r_{2}(x, y)}\right)^{n}}{r_{1}(x, y)-r_{2}(x, y)} \\
& =\frac{p(x, y)\left(\frac{r_{2}(x, y)}{2 x}\right)^{n}-q(x, y)\left(\frac{r_{1}(x, y)}{2 x}\right)^{n}}{r_{1}(x, y)-r_{2}(x, y)} \\
& =\frac{1}{(2 x)^{n}}\left(\frac{p(x, y) r_{2}(x, y)-q(x, y) r_{1}(x, y)^{n}}{r_{1}(x, y)-r_{2}(x, y)}\right) .
\end{aligned}
$$

We will define the following matrix $Q B_{n}(x, y)=\left(\begin{array}{cc}-2 x Q M_{n-1}(x, y) & Q M_{n}(x, y) \\ -2 x Q M_{n}(x, y) & Q M_{n+1}(x, y)\end{array}\right)$.

Next, we show that it can be generated by the product of matrices such as:

$$
\left(\begin{array}{cc}
0 & 1 \\
-2 x .1 & 3 y
\end{array}\right)^{n}\left(\begin{array}{cc}
-2 x Q M_{-1}(x, y) & Q M_{0}(x, y) \\
-2 x Q M_{0}(x, y) & Q M_{1}(x, y)
\end{array}\right)
$$

for every integer $n \geqslant 0$. 
Theorem 4.3. For every integer $n \geqslant 0$, we have:

(i) $Q B_{n}(x, y)=\left(\begin{array}{cc}-2 x Q M_{n-1}(x, y) & Q M_{n}(x, y) \\ -2 x Q M_{n}(x, y) & Q M_{n+1}(x, y)\end{array}\right)=\left(\begin{array}{cc}0 & 1 \\ -2 x .1 & 3 y\end{array}\right)^{n}\left(\begin{array}{cc}-2 x Q M_{-1}(x, y) & Q M_{0}(x, y) \\ -2 x Q M_{0}(x, y) & Q M_{1}(x, y)\end{array}\right)$;

(ii) $\operatorname{det}\left(Q B_{n}(x, y)\right)=-(2 x)^{n+1}\left(Q M_{-1}(x, y)-Q M_{0}(x, y)^{2}\right)$;

(iii) $Q B_{-1}(x, y)=\left(\begin{array}{cc}-2 x Q M_{-n-1}(x, y) & Q M_{-n}(x, y) \\ -2 x Q M_{-n}(x, y) & Q M_{-n+1}(x, y)\end{array}\right)$

$=\left(\begin{array}{cc}0 & 1 \\ -2 x .1 & 3 y\end{array}\right)^{-n}\left[\left(\begin{array}{ll}1 & 0 \\ 0 & 1\end{array}\right)+\left(\begin{array}{cc}\frac{3 y}{2 x} & -\frac{1}{2 x} \\ 1 & 0\end{array}\right) i+\left(\begin{array}{cc}-\frac{\left(2 x-9 y^{2}\right)}{4 x^{2}} & -\frac{3 y}{4 x^{2}} \\ \frac{3 y}{2 x} & -\frac{1}{2 x}\end{array}\right) j+\left(\begin{array}{cc}-\frac{\left(27 y^{3}-4 x y\right)}{8 x^{3}} & \frac{\left(2 x-9 y^{2}\right)}{8 x^{3}} \\ -\frac{\left(2 x-9 y^{2}\right)}{4 x^{2}} & -\frac{3 y}{4 x^{2}}\end{array}\right) k\right]$.

Proof. We consider the matrix:

$$
\begin{aligned}
& Q B_{n}(x, y)\left(\begin{array}{cc}
-2 x Q M_{n-1}(x, y) & Q M_{n}(x, y) \\
-2 x Q M_{n}(x, y) & Q M_{n+1}(x, y)
\end{array}\right) \\
& =\left(\begin{array}{cc}
-2 x\left(M_{n-1}(x, y)+M_{n}(x, y) i+M_{n+1}(x, y) j+M_{n+2}(x, y) k\right) & M_{n}(x, y)+M_{n+1}(x, y) i+M_{n+2}(x, y) j+M_{n+3}(x, y) k \\
-2 x\left(M_{n}(x, y)+M_{n+1}(x, y) i+M_{n+2}(x, y) j+M_{n+3}(x, y) k\right) & M_{n+1}(x, y)+M_{n+2}(x, y) i+M_{n+3}(x, y) j+M_{n+4}(x, y) k
\end{array}\right) \\
& =\left(\begin{array}{cc}
-2 x M_{n-1}(x, y) & M_{n}(x, y) \\
-2 x M_{n}(x, y) & M_{n+1}(x, y)
\end{array}\right)+\left(\begin{array}{cc}
-2 x M_{n}(x, y) & M_{n+1}(x, y) \\
-2 x M_{n+1}(x, y) & M_{n+2}(x, y)
\end{array}\right) i \\
& +\left(\begin{array}{ll}
-2 x M_{n+1}(x, y) & M_{n+2}(x, y) \\
-2 x M_{n+2}(x, y) & M_{n+3}(x, y)
\end{array}\right) j+\left(\begin{array}{ll}
-2 x M_{n+2}(x, y) & M_{n+3}(x, y) \\
-2 x M_{n+3}(x, y) & M_{n+4}(x, y)
\end{array}\right) k \\
& =\left(\begin{array}{cc}
0 & 1 \\
-2 x .1 & 3 y
\end{array}\right)^{n}+\left(\begin{array}{cc}
0 & 1 \\
-2 x .1 & 3 y
\end{array}\right)^{n+1} i+\left(\begin{array}{cc}
0 & 1 \\
-2 x .1 & 3 y
\end{array}\right)^{n+2} j+\left(\begin{array}{cc}
0 & 1 \\
-2 x .1 & 3 y
\end{array}\right)^{n+3} k \\
& =\left(\begin{array}{cc}
0 & 1 \\
-2 x .1 & 3 y
\end{array}\right)^{n}\left[\left(\begin{array}{cc}
1 & 0 \\
0 & 1
\end{array}\right)+\left(\begin{array}{cc}
0 & 1 \\
-2 x .1 & 3 y
\end{array}\right) i+\left(\begin{array}{cc}
0 & 1 \\
-2 x .1 & 3 y
\end{array}\right)^{2} j+\left(\begin{array}{cc}
0 & 1 \\
-2 x .1 & 3 y
\end{array}\right)^{3} k\right] \\
& =\left(\begin{array}{cc}
0 & 1 \\
-2 x .1 & 3 y
\end{array}\right)^{n}\left[\left(\begin{array}{cc}
-2 x M_{-1}(x, y) & M_{0}(x, y) \\
-2 x M_{0}(x, y) & M_{1}(x, y)
\end{array}\right)+\left(\begin{array}{cc}
-2 x M_{0}(x, y) & M_{1}(x, y) \\
-2 x M_{1}(x, y) & M_{2}(x, y)
\end{array}\right) i+\left(\begin{array}{cc}
-2 x M_{1}(x, y) & M_{2}(x, y) \\
-2 x M_{2}(x, y) & M_{3}(x, y)
\end{array}\right) j\right] \\
& +\left(\begin{array}{cc}
0 & 1 \\
-2 x .1 & 3 y
\end{array}\right)^{n}\left[\left(\begin{array}{ll}
-2 x M_{2}(x, y) & M_{3}(x, y) \\
-2 x M_{3}(x, y) & M_{4}(x, y)
\end{array}\right) k\right] \\
& =\left(\begin{array}{cc}
0 & 1 \\
-2 x .1 & 3 y
\end{array}\right)^{n}\left(\begin{array}{cc}
-2 x\left(-\frac{1}{2 x}+0 i+1 j+3 y k\right) & 0+i+3 y j+\left(9 y^{2}-2 x\right) k \\
-2 x\left(0+i+3 y j+\left(9 y^{2}-2 x\right) k\right) & 1+3 y i+\left(9 y^{2}-2 x\right) j+\left(27 y^{3}-12 x y\right) k
\end{array}\right) \\
& =\left(\begin{array}{cc}
0 & 1 \\
-2 x .1 & 3 y
\end{array}\right)^{n}\left(\begin{array}{cc}
-2 x Q M_{-1}(x, y) & Q M_{0}(x, y) \\
-2 x Q M_{0}(x, y) & Q M_{1}(x, y)
\end{array}\right) \text {. }
\end{aligned}
$$

Finally, we will take the matrix, for every $n \geqslant 1$, we have:

$$
\begin{aligned}
& Q B_{-n}(x, y)=\left(\begin{array}{cc}
-2 x Q M_{-n-1}(x, y) & Q M_{-n}(x, y) \\
-2 x Q M_{-n}(x, y) & Q M_{-n+1}(x, y)
\end{array}\right) \\
& =\left(\begin{array}{cc}
-2 x\left(M_{-n-1}(x, y)+M_{-n}(x, y) i+M_{-n+1}(x, y) j+M_{-n+2}(x, y) k\right) & M_{-n}(x, y)+M_{-n+1}(x, y) i+M_{-n+2}(x, y) j+M_{-n+3}(x, y) k \\
-2 x\left(M_{-n}(x, y)+M_{-n+1}(x, y) i+M_{-n+2}(x, y) j+M_{-n+3}(x, y) k\right) & M_{-n+1}(x, y)+M_{-n+2}(x, y) i+M+n+3 \\
-n, y) j+M_{-n+4}(x, y) k
\end{array}\right) \\
& =\left(\begin{array}{cc}
-2 x M_{-n-1}(x, y) & M_{-n}(x, y) \\
-2 x M_{-n}(x, y) & M_{-n+1}(x, y)
\end{array}\right)+\left(\begin{array}{cc}
-2 x M_{-n}(x, y) & M_{-n+1}(x, y) \\
-2 x M_{-n+1}(x, y) & M_{-n+2}(x, y)
\end{array}\right)+\left(\begin{array}{cc}
-2 x M_{-n+1}(x, y) & M_{-n+2}(x, y) \\
-2 x M_{-n+2}(x, y) & M_{-n+3}(x, y)
\end{array}\right) j \\
& +\left(\begin{array}{ll}
-2 x M_{-n+2}(x, y) & M_{-n+3}(x, y) \\
-2 x M_{-n+3}(x, y) & M_{-n+4}(x, y)
\end{array}\right) k
\end{aligned}
$$




$$
\begin{aligned}
& =\left(B_{-n}(x, y)+B_{-n+1}(x, y) i+B_{-n+2}(x, y) j+B_{-n+3}(x, y) k\right) \\
& =\left(B_{-n}(x, y)+B_{-(n-1)}(x, y) i+B_{-(n-2)}(x, y) j+B_{-(n-3)}(x, y) k\right) \\
& =\left(\begin{array}{cc}
0 & 1 \\
-2 x .1 & 3 y
\end{array}\right)^{-n}+\left(\begin{array}{cc}
0 & 1 \\
-2 x .1 & 3 y
\end{array}\right)^{-n-1} i+\left(\begin{array}{cc}
0 & 1 \\
-2 x .1 & 3 y
\end{array}\right)^{-n-2} j+\left(\begin{array}{cc}
0 & 1 \\
-2 x .1 & 3 y
\end{array}\right)^{-n-3} k \\
& =\left(\begin{array}{cc}
0 & 1 \\
-2 x .1 & 3 y
\end{array}\right)^{-n}\left[\left(\begin{array}{ll}
1 & 0 \\
0 & 1
\end{array}\right)+\left(\begin{array}{cc}
0 & 1 \\
-2 x .1 & 3 y
\end{array}\right)^{-1} i+\left(\begin{array}{cc}
0 & 1 \\
-2 x .1 & 3 y
\end{array}\right)^{-2} j+\left(\begin{array}{cc}
0 & 1 \\
-2 x .1 & 3 y
\end{array}\right)^{-3} k\right] \\
& =\left(\begin{array}{cc}
0 & 1 \\
-2 x .1 & 3 y
\end{array}\right)^{-n}\left[\left(\begin{array}{ll}
1 & 0 \\
0 & 1
\end{array}\right)+\left(\begin{array}{cc}
\frac{3 y}{2 x} & -\frac{1}{2 x} \\
1 & 0
\end{array}\right) i+\left(\begin{array}{cc}
-\frac{\left(2 x-9 y^{2}\right)}{4 x^{2}} & -\frac{3 y}{4 x^{2}} \\
\frac{3 y}{2 x} & -\frac{1}{2 x}
\end{array}\right) j+\left(\begin{array}{cc}
-\frac{\left(27 y^{3}-4 x y\right)}{8 x^{3}} & \frac{\left(2 x-9 y^{2}\right)}{8 x^{3}} \\
-\frac{\left(2 x-9 y^{2}\right)}{4 x^{2}} & -\frac{3 y}{4 x^{2}}
\end{array}\right) k\right] \text {, }
\end{aligned}
$$

from where the result follows.

Finally, let us see the corresponding generating function with the notion of generating function for the bivariate Mersenne quaternion.

Theorem 4.4. The generating function for the bivariate Mersenne polynomials is:

$$
G Q M(t)=\frac{Q M_{0}(x, y)+\left(Q M_{1}(x, y)-3 y Q M_{0}(x, y)\right) t}{1-3 y t+2 x t^{2}} .
$$

Proof. We consider the formal series indicated by $G Q M(t) \sum_{i=0}^{\infty} Q M_{i}(x, y) t^{i}$, where the coefficients are exactly the quaternions bivariate polynomials of Mersenne.

We observe, however, the following relationship

$$
Q M_{n+2}(x, y)-3 y Q M_{n+1}(x, y)+2 x Q M_{n}=0, \forall n \geqslant 0 .
$$

In this way, we will consider the following expressions constituted as infinite sums indicated below:

$$
\begin{aligned}
1 G Q M(t) & =Q M_{0}(x, y) t^{0}+Q M_{1}(x, y) t+\ldots+Q M_{n}(x, y) t^{n}+\ldots \\
(-3 y) G Q M(t) t & =-3 y Q M_{0}(x, y) t-3 y Q M_{1}(x, y) t^{2}-\ldots-3 y Q M_{n-1}(x, y) t^{n}+\ldots \\
(2 x) G Q M(t) t^{2} & =2 x Q M_{0}(x, y) t^{2}+2 x y Q M_{1}(x, y) t^{3}+\ldots+2 x Q M_{n-2}(x, y) t^{n}+\ldots
\end{aligned}
$$

Then, we group the expression:

$$
\begin{aligned}
& G Q M(t)-3 y G Q M(t) t+2 x G Q M(t) t^{2} \\
&= G Q M(t)\left(1-3 y t+2 x t^{2}\right) \\
&= Q M_{0}(x, y)+\left(Q M_{1}(x, y)-3 y Q M_{0}(x, y)\right) t+\left(Q M_{2}(x, y)-3 y Q M_{1}(x, y)+2 x Q M_{0}(x, y)\right) t^{2} \\
&+0 t^{3}+\ldots+\left(Q M_{n}(x, y)-3 y Q M_{n-1}(x, y)+2 x Q M_{n-2}(x, y)\right) t^{n}+\ldots
\end{aligned}
$$

Finally, we will find that:

$$
\begin{aligned}
G Q M(t) & =\frac{Q M_{0}(x, y)+\left(Q M_{1}(x, y)-3 y Q M_{0}(x, y)\right) t}{1-3 y t+2 x t^{2}} \\
& =\frac{i+3 y j+\left(1+(3 y-3) i+\left(9 y^{2}-9 y-2 x\right) j+\left(27 y^{3}-12 x y\right) k\right) t}{1-3 y t+2 x t^{2}} .
\end{aligned}
$$




\section{Conclusion}

In this work we present a new notion called bivariate Mersenne polynomials and some generating matrices. Some generalized results can be found in the work [3], however, the properties presented in the previous sections involve matrix properties.

\section{Acknowledgments}

We are grateful for the financial support granted in Brazil for the development of this research by the National Council for Scientific and Technological Development.

\section{References}

[1] Catarino, P. M. M. C., Campos, H., \& Vasco, P. (2016). On the Mersenne sequence, Annales Mathematicae et Informaticae, 1 (46), 36-53.

[2] Catalani, M. (2002). Generalized Bivariate Fibonacci Polynomials, Arxiv, 1-11.

[3] Dasdemir, A., \& Bilgici, G. (2019). Gaussian Mersenne numbers and generalized Mersenne quaternions, Notes on Number Theory and Discrete Mathematics, 25 (3), 87-96. 